\title{
Mental health status and work environment among workers in small- and medium-sized enterprises in Guangdong, China-a cross-sectional survey
}

Zhi Zeng ${ }^{1,2 \dagger}$, Yan Guo ${ }^{1 \dagger}$, Liming Lu', Lu Han ${ }^{1}$, Wen Chen ${ }^{1}$ and Li Ling ${ }^{1 *}$

\begin{abstract}
Background: Small- and medium-sized enterprises (SMEs) generate nearly 80\% of the jobs in China, but the dangerous work environment often found in these enterprises poses a major concern for public health. Psychosocial pressure and mental health problems among the workers are also common in SMEs. However, mental health of workers in SMEs is largely neglected in occupational health research and practice in China. The purpose of this study is to assess mental health of the workers and to explore the associations between physical and psychosocial work environment and workers' mental health in SMEs in South China.
\end{abstract}

Methods: Data were collected in 2012 through a cross-sectional survey among 1200 workers working in small- and medium-sized enterprises (SMEs) in Guangdong, China. Mental health was measured by psychological well-being in the current study. Job Demand-Control-Support (JDCS) model was used as a theoretical framework to examine the psychosocial factors associated with workers' psychological well-being. Data were analyzed using SPSS 20.0 and analysis was performed using bivariate analyses and multivariate logistic regression.

Results: About three in ten workers (35.3\%) in the sample had poor psychological well-being. Those who were men, younger in age, or migrant workers had worse psychological outcome in bivariate analyses. After controlling for individual variables (gender, age, marital status, and household registration), we found that longer weekly work hours $(\mathrm{OR}=1.30,95 \% \mathrm{Cl}: 1.13 \sim 1.50)$, more exposure to hazardous work environment $(\mathrm{OR}=1.26,95 \% \mathrm{Cl}: 1.10 \sim 1.44)$, higher job demands $(\mathrm{OR}=1.29,95 \% \mathrm{Cl}: 1.12 \sim 1.49)$, and lower job autonomy (OR=0.70, 95\% Cl: $0.60 \sim 0.81)$ were significant associated with worse psychological well-being. The results were consistent with predictions of the JDCS model.

Conclusions: The results indicate that the JDCS model is a useful framework in predicting psychological well-being among Chinese workers in SMEs. Future mental health promotion should focus on young migrant male workers as they appear to be most vulnerable in their psychological well-being. Both physical and psychosocial aspects of the work environment should be taken into account in policy making to prevent mental disorder and promote psychological well-being among workers in SMEs.

Keywords: Chinese workers, Mental health, Psychological well-being, WHO (five) well-being index, Small- and medium-sized enterprises (SMEs), Job demand-control (JDC) model, Job demand-control-support (JDCS) model

\footnotetext{
* Correspondence: lingli@mail.sysu.edu.cn

${ }^{\dagger}$ Equal contributors

'Sun Yat-sen Center for Migrant Health Policy, School of Public Health,

SunYat-sen University, Guangzhou 510405, China

${ }^{2}$ Maternal and Child Health Hospital of Hunan Province, Changsha 410008, China
} 


\section{Background}

China has experienced dramatic industrialization, urbanization, and economic growth over the last three decades. Small- and medium-sized enterprises (SMEs) play a crucial role in the Chinese economy, providing about $80 \%$ of the jobs in urban China [1]. Compared to large enterprises, SMEs tend to be less regulated in safe work environment. In addition, employees in SMEs usually have low wages and lack of effective long-term occupational health services [2]. Rural-to-urban migrants consist of the majority of the employees in SMEs who migrate from less developed areas to more developed areas in China [3]. It was estimated that the number of migrant workers had reached 263 million (19.4\% of the total population) in 2012 [4]. Compared to local residents, mental health problems and suicides are more likely to occur in these migrants [5]. Studies have shown that mental health of the workers in SMEs is worse than both the general population and those in large enterprises [6,7]. However, mental health of the workers in SMEs is largely neglected in existing occupational health research and practice in China [8].

It is estimated that the burden of mental illness will account for $1 / 4$ of the total disease burden in 2020 globally [9]. Mental health problems are common in the Chinese working population. Depression is one of the most disabling of mental disorders. According to the Chinese Health Education Center Survey, about 50\% of the 13,177 workers sampled in 6 provinces or municipalities had symptoms of depression [10]. The definition of mental health is not just the occurrence of mental disorder, but also a state of absence of well-being, from the perspective of primary prevention [11]. Literature suggests that decreased well-being is the first sign of distress $[12,13]$.

Working conditions are key to understanding psychological well-being of workers who are frequently exposed to hazardous environment. Both physical and psychosocial work environment are important. According to previous studies, shift work, long work hours, hazardous work environment (e.g., exposure to noise or toxic chemicals) might be potential risk factors for poor mental health of the Chinese workers [14-17]. Research has also shown a close relationship between psychosocial workrelated factors and mental health of workers [18]. Such relationship, however, is largely neglected in the existing literature on workers in China.

Over the past several decades, one of the most influential models in examining the relationship between work environment and mental health is the Job Demand-ControlSupport (JDCS) model. The JDCS model identifies three critical aspects at work: job demands, job autonomy and worksite support [19]. A recent meta-analysis reveals that high job demands and low job autonomy are risk factors for poor mental health and that the psychosocial aspect of the work environment is important for workers' mental health [20]. However, there are few empirical studies utilizing the JDCS model and considering both physical and psychosocial work-related factors on the mental health of the SMEs workers in China.

The purposes of the current study are to assess the mental health status of the SMEs workers, and to explore the associated work-related factors (both physical and psychosocial) with the mental health status of the SMEs workers in South China.

\section{Methods}

\section{Study setting}

This cross-sectional study was conducted between September and October in 2012 in Guangdong Province, China. A two-stage stratified random sampling method was carried out. First, two developed cities (Guangzhou and Foshan) and two less-developed cities (Zhaoqing and Qingyuan) in Guangdong Province were selected. Second, based on the final sample size requirement (see next section for detail), we randomly selected SMEs in shoe making, electronics, and plastics industries from each city by applying computer-generated random numbers from the list of enterprises in these cities. The reason to choose industries in shoe making, electronics, and plastics is that workers in these industries were exposed to the same occupational hazard, organic solvent, which causes about $50 \%$ of all cases related to occupational diseases in Guangdong Province [21]. The county Centers for Disease Control and Prevention (CDC) (or occupational health institute) assisted in the selection process and encouraged the selected local enterprises to participate in the study. Finally, front-line workers in assembly lines were recruited from the sampled SMEs for participation.

\section{Sample size estimation}

According to literature review, the rate of depression among occupational group was $50 \%$ in China [10]. Assuming 95\% confidence level and an absolute sampling error of $4 \%$, a minimum of 600 workers are needed. From literature and our previous experience, taking into account of the $70 \%$ of response rate and $80 \%$ of completion rate, the needed sample size was 1071 .

\section{Study Subjects}

The recruitment criteria for workers included: 1) frontline workers in assembly lines who were exposed to occupational hazards (noise, or toxic chemicals); 2) workers who had resided in the study area for at least six months. Exclusion criteria included team leaders or management personnel who differ in their work environmental exposure [22]. Workers completed the questionnaire 
individually. Trained investigators provided assistance to participants whenever needed. Each questionnaire was checked by two investigators for missing data or logical inconsistency. It typically took about 20 minutes to finish the survey. A small monetary incentive (RMB 20, equal USD 3.26) was provided for participants upon their completion of the survey. Finally, data from 1200 workers (response rate, 90\%) in 10 enterprises were analyzed.

\section{Ethics statement}

The study was approved by the Institutional Review Board (IRB) of the School of Public Health, Sun Yat-sen University in China. Written informed consents were obtained from all study participants.

\section{Measurements}

\section{WHO well-being scale}

We employed psychological well-being to examine mental health status of workers in SMEs in China. The World Health Organization (WHO) (five) Well-Being Index, 1998 version (WHO-5) [23] was designed for measuring psychological well-being. It is the short form (five items) of the earlier versions of this instrument [24]. The WHO-5 is recommended by the WHO as the first step in a two-stage screening process for depression and should be followed by a clinician interview [25]. The diagnostic validity of the WHO-5 in the screening of depression is good [Area Under the Curve (AUC) value $=0.88]$, as indicated by several studies [26-28].

WHO-5 is a uni-dimensional measure that asks the feelings of participants in the previous two weeks and contains five positively-worded items: "I have been cheerful and in good spirits"; "I have been calm and relaxed"; "I have been active and vigorous"; "When I wake up, I feel fleshed and rested"; and "My daily life is filled with things that interest me". The options of the answers regarding their feelings are on a 6-point likert scale ranging from 0 (not present at all) to 5 (constantly present). The possible total scores vary from 0 to 25 , with higher scores indicating better well-being. Scores of 13 and below indicate poor well-being and warrant follow-up diagnostic procedures $[26,29,30]$.

\section{Physical work conditions}

Physical work conditions were measured by whether workers had employment contract (yes/no) and shift work schedule (yes/no), how many work hours per week, and physical work environment which was assessed by two questions:" How often are you exposed to disturbing noise?" "How often are you exposed to excessive toxic chemicals?" Responses for the two exposure questions were on a five-point scale ranging from 1 (not at all) to 5 (almost all the time).

\section{Psychosocial work conditions}

According to the Job Demand-Control-Support (JDCS) model [19], and Chinese (Mainland) Version of Job Content Questionnaire [31], psychosocial work conditions were measured by three dimensions, including job demands, job autonomy, and worksite support. Job demands referred to work load, and had been operationalized mainly in terms of time pressure and role conflict. Job autonomy referred to a person's ability to control his or her work activities. Worksite support included support from supervisors and coworkers. Responses were on a five-point scale ranging from 1 (strongly disagree) to 5 (strongly agree). Please see the details of the JDCS model construct items in Table 1.

\section{Individual characteristics}

Participants also provided individual characteristics on gender, age, educational attainment (primary school or lower, junior high school, senior high school, college or higher), individual monthly income, household registration (permanent residents or migrant workers).

\section{Statistical analyses}

A database was constructed using Epidata 3.0 and statistical analyses were performed using SPSS version 20.0. First, as part of a test of reliability, internal consistency was examined using Cronbach's alpha. WHO (five) Well-Being Index, extent of exposure to hazardous environment, job demands, job autonomy, and worksite support demonstrated a reliability alpha coefficient of $0.91,0.70,0.70,0.73,0.61$, respectively, suggesting that all of the measures had acceptable to satisfactory internal reliability [32].

The WHO (five) Well Being Index scale scores were calculated according to the established algorithms with higher scores indicating better mental health status.

Table 1 The JDCS Model Construct Items

\begin{tabular}{|c|c|c|c|c|}
\hline Construct & Item & Item text & Range & $\begin{array}{c}\text { Cronbach's } \\
\text { Alpha }\end{array}$ \\
\hline Job demands & 1 & $\begin{array}{l}\text { My job requires working } \\
\text { very fast. } \\
\text { My job requires working } \\
\text { very hard. }\end{array}$ & $2 \sim 10$ & 0.70 \\
\hline Job autonomy & 2 & $\begin{array}{l}\text { I have enough resources } \\
\text { to manage my job. } \\
\text { At my workplace I dare } \\
\text { to speak my minds. } \\
\text { I have the freedom to } \\
\text { decide how my tasks are } \\
\text { to be carried out. }\end{array}$ & $3 \sim 15$ & 0.73 \\
\hline Worksite support & 1 & $\begin{array}{l}\text { I get along well with } \\
\text { my supervisors. } \\
\text { I get along well with } \\
\text { my coworkers. }\end{array}$ & $2 \sim 10$ & 0.61 \\
\hline
\end{tabular}


Well-being scores were categorized into two categories $(1 \sim 13 / 14 \sim 25)$. The distribution of individual characteristics and work-related factors were illustrated by numbers and percentages, means and standard deviations as appropriate. Bivariate analyses were employed to analyze the associations between factors and mental health. Multivariate logistic regressions were used to simultaneously identify work-related variables associated with mental health, while adjusting for individual characters significant in bivariate analyses at $P<0.05$. Odds ratios (ORs) with 95\% confidence interval (CI) were presented. Two-sided tests of significance and confidence intervals were based on the 0.05 level.

\section{Results}

\section{Characteristics of the study population}

Table 2 presents individual and work-related characteristics of the workers as well as their mental health status. Most of the workers were male (54.4\%), being married or co-habiting (60.4\%). The average age was 31.1 years. Majority of the participants were migrant workers (75.7\%) and had signed employment contract (92.8\%); about two fifths $(39.1 \%)$ worked on a shift work schedule, with average work hours of 55.4 per week and monthly income of 2431RMB (USD 396) (The average monthly wage of workers was 3762 RMB (USD 617) in urban areas of Guangdong Province in 2012) [33]. About one third $(35.3 \%)$ of them reported poor psychological well-being in the past two weeks.

\section{Comparative analyses}

Summaries of the bivariate analyses are presented in Table 3. Those who were men, younger in age, or migrant workers were more likely to have worse psychological well-being than their counterparts. Educational level, individual monthly income, shift work schedule were not significantly associated with psychological wellbeing. More exposure to hazardous work environment, higher job demands, and lower job autonomy were risk factors for psychological well-being. Workers with longer work hours per week had worse psychological wellbeing, and those who signed a contract with enterprises had better psychological well-being.

\section{Multivariate logistic regression}

Table 4 presents the associated factors with mental health of participants in multivariate logistic analyses. Dependent variable was poor psychological well-being $(0 \sim 13=1,14 \sim 25=0)$. After controlling for individual characters (gender, age, marital status, and household registration) significant in bivariate analyses at $P<0.05$, longer weekly work hours $(\mathrm{OR}=1.30,95 \% \mathrm{CI}: 1.13 \sim 1.50)$, more exposure to hazardous work environment $(\mathrm{OR}=1.26$, $95 \%$ CI: $1.10 \sim 1.44$ ) were significantly and negatively
Table 2 Individual and work-related characteristics and mental health of the study population $(N=1200)$

\begin{tabular}{|c|c|}
\hline Variables & $N(\%)$ \\
\hline \multicolumn{2}{|l|}{ Individual characteristics } \\
\hline Age (years) (Mean $\pm S D$ ) & $31.1 \pm 9.4$ \\
\hline \multicolumn{2}{|l|}{ Gender } \\
\hline Men & $653(54.4)$ \\
\hline Women & $547(45.6)$ \\
\hline \multicolumn{2}{|l|}{ Marital status } \\
\hline Married or co-habiting & $727(60.5)$ \\
\hline Single & 464(38.7) \\
\hline Divorced & $9(0.8)$ \\
\hline \multicolumn{2}{|l|}{ Education level } \\
\hline Primary school or lower & $79(6.6)$ \\
\hline Junior high school & $587(48.7)$ \\
\hline Senior high school & $443(37.1)$ \\
\hline College or higher & $91(7.6)$ \\
\hline Individual monthly income (yuan) (Mean $\pm S D$ ) & $2431 \pm 937$ \\
\hline \multicolumn{2}{|l|}{ Household registration } \\
\hline Permanent resident workers & 293(24.3) \\
\hline Migrant workers & $907(75.7)$ \\
\hline \multicolumn{2}{|l|}{ Physical work conditions } \\
\hline \multicolumn{2}{|l|}{ Employment contract } \\
\hline Yes & 1114(92.8) \\
\hline No & $86(7.2)$ \\
\hline \multicolumn{2}{|l|}{ Shift work schedule } \\
\hline Yes & $466(39.1)$ \\
\hline No & 734(60.9) \\
\hline Work hours per week (hours) (Mean $\pm S D$ ) & $55.4 \pm 8.1$ \\
\hline Extent of exposure to hazardous environment (Mean $\pm S D$ ) & $5.5 \pm 2.4$ \\
\hline \multicolumn{2}{|l|}{ Psychosocial work conditions } \\
\hline Job demands (Mean $\pm S D$ ) & $4.4 \pm 1.5$ \\
\hline Job autonomy (Mean \pm SD) & $10.5 \pm 2.3$ \\
\hline Worksite support (Mean \pm SD) & $7.5 \pm 1.1$ \\
\hline \multicolumn{2}{|l|}{ Mental health } \\
\hline \multicolumn{2}{|l|}{ Psychological well-being } \\
\hline $0 \sim 13$ & $424(35.3)$ \\
\hline $14 \sim 25$ & $776(64.7)$ \\
\hline
\end{tabular}

associated with poor psychological well-being. Higher job demands $(\mathrm{OR}=1.29,95 \% \mathrm{CI}: 1.12 \sim 1.49)$, and lower job autonomy $(\mathrm{OR}=0.70,95 \% \mathrm{CI}: 0.60 \sim 0.81)$ were significantly and negatively associated with poor psychological well-being. The results were consistent with the JDCS model as the model predicts psychosocial factors associated with mental health status of workers in SMEs [19]. 
Table 3 Bivariate analyses showing association of individual and work-related factors with mental health status of workers in SMEs $(N=1200)$

\begin{tabular}{|c|c|c|}
\hline \multirow{3}{*}{ Variables } & \multicolumn{2}{|c|}{$\begin{array}{l}\text { Poor psychological } \\
\text { well-being }\end{array}$} \\
\hline & $0 \sim 13$ & $14 \sim 25$ \\
\hline & $(N=424,35.3 \%)$ & $(N=776,64.7 \%)$ \\
\hline \multicolumn{3}{|l|}{ Individual characteristics } \\
\hline Age (years) (Mean $\pm S D$ ) & $28.6 \pm 8.7^{* *}$ & $32.4 \pm 9.6$ \\
\hline \multicolumn{3}{|l|}{ Gender } \\
\hline Men & $265(40.2)^{* *}$ & $388(59.8)$ \\
\hline Women & 159(29.6) & $388(70.4)$ \\
\hline \multicolumn{3}{|l|}{ Marital status } \\
\hline Married/co-habiting & $201(28.3)^{* *}$ & $524(71.7)$ \\
\hline Single & $220(46.3)$ & 244(53.7) \\
\hline Divorced & $3(33.3)$ & $6(66.7)$ \\
\hline \multicolumn{3}{|l|}{ Educational level } \\
\hline Primary school or lower & $25(32.5)$ & $54(67.5)$ \\
\hline Junior high school & 202(33.9) & $385(66.1)$ \\
\hline Senior middle school & 163(37.1) & $280(62.9)$ \\
\hline College or higher & $34(37.4)$ & $57(62.6)$ \\
\hline $\begin{array}{l}\text { Individual monthly income } \\
\text { (yuan) (Mean } \pm S D \text { ) }\end{array}$ & $2350 \pm 855$ & $2462 \pm 826$ \\
\hline \multicolumn{3}{|l|}{ Household registration } \\
\hline Permanent resident workers & $84(29.0)^{* *}$ & 209(71.0) \\
\hline Migrant workers & $340(37.4)$ & $567(62.6)$ \\
\hline \multicolumn{3}{|l|}{ Physical work conditions } \\
\hline \multicolumn{3}{|l|}{ Employment contract } \\
\hline Yes & $386(34.5)^{*}$ & $728(65.5)$ \\
\hline No & $38(45.7)$ & $48(54.3)$ \\
\hline \multicolumn{3}{|l|}{ Shift work schedule } \\
\hline Yes & 179(38.5) & $287(61.5)$ \\
\hline No & $245(33.3)$ & $489(66.7)$ \\
\hline $\begin{array}{l}\text { Work hours per week (hours) } \\
\text { (Mean } \pm \text { SD) }\end{array}$ & $56.8 \pm 7.9^{* *}$ & $54.6 \pm 8.0$ \\
\hline $\begin{array}{l}\text { Extent of exposure to hazardous } \\
\text { environment (Mean } \pm S D \text { ) }\end{array}$ & $5.8 \pm 2.3^{* *}$ & $5.3 \pm 2.3$ \\
\hline \multicolumn{3}{|l|}{ Psychosocial work conditions } \\
\hline Job demands (Mean \pm SD) & $4.8 \pm 1.4^{* *}$ & $4.2 \pm 1.5$ \\
\hline Job autonomy (Mean $\pm S D)$ & $9.8 \pm 2.4^{* *}$ & $10.9 \pm 2.2$ \\
\hline Worksite support (Mean \pm SD) & $7.4 \pm 1.1$ & $7.5 \pm 1.0$ \\
\hline
\end{tabular}

${ }^{*} P<0.05,{ }^{*} P<0.01$.

\section{Discussion}

The current study intended to assess mental health of workers in SMEs in Guangdong, China and to explore the related physical and psychosocial factors of the work environment. $35.3 \%$ of the workers were found poor in their psychological well-being status. Those who were men, younger in age, or migrant workers were more
Table 4 Multivariate logistic regression analyses showing association of individual and work-related factors with mental health status of workers in SMEs $(N=1200)$

\begin{tabular}{cc}
\hline & $\begin{array}{c}\text { Poor psychological } \\
\text { well-being } \\
\text { Variables } \\
\end{array}$ \\
& $(0 \sim 13=1 / 14 \sim 25=0)$ \\
OR $(95 \% \mathrm{Cl})$
\end{tabular}

\section{Individual characteristics}

Age (years)

$0.74(0.61 \sim 0.89)^{* *}$

Gender

Men (reference)

Women

$0.61(0.46 \sim 0.82)^{* *}$

Marital status

Married/co-habiting (reference)

Single

$0.77(0.54 \sim 1.11)$

Divorced

$1.18(0.28 \sim 5.09)$

Household registration

Permanent resident workers (reference)

Migrant workers

$1.36(1.01-1.85)^{*}$

Physical work conditions

Employment contract

Yes (reference)

No

$1.04(0.62-1.73)$

Shift work

No (reference)

Yes

$0.86(0.63-1.17)$

Work hours per week

Extent of exposure to hazardous environment

$1.30(1.13-1.50)^{* * *}$

Psychosocial work conditions

Job demands

$1.29(1.12-1.49)^{* *}$

Job autonomy

$0.70(0.60-0.81)^{* * *}$

Worksite support

$0.97(0.85-1.11)$

${ }^{*} P<0.05,{ }^{* *} P<0.01,{ }^{* * *} P<0.001$.

Adjusting for individual characters (gender, age, marital status, and household registration) significant in bivariate analyses at $P<0.05$.

likely to have worse psychological well-being. Our study found significant associations between physical work-related factors (i.e., weekly work hours, extent of exposure to hazardous work environment) and mental health. In addition, we also found significant associations between psychosocial work factors (i.e., job demands and job autonomy) and mental health among workers in SMEs, guided by the Job Demand-ControlSupport (JDCS) model [19].

Results of the current study are consistent with the predictions of the JDCS model (higher job demands and lower job autonomy were significantly and negatively associated with mental health). Such model has been widely applied to mental health and psychological wellbeing research $[34,35]$. The current study is the first 
effort to apply the JDCS model to examine the mental health of Chinese workers in SMEs. Results of the current study indicate that the JDCS model is a useful framework predicting mental health among workers in SMEs in South China.

Even though the New Labor Law was passed in 2008 in China that regulated no more than 40 hours a week among workers, such regulation was not strictly enforced. In the current study, the average weekly work hours were 55.4 hours. Previous study suggests a negative association between work hours and workers' mental status, with odds ratio being 2-4 for depression for those working more than 40 hours per week [36]. In our study, work hours is also a significant indicator for workers' mental health. All the findings indicate the importance of reinforcing the regulations of the New Labor Law on maximum work hours for factory workers. Similar to other studies, results show that more exposure to hazardous work environment increases the risk of mental health problems among Chinese workers in SMEs [7,37]. Workers exposed to physical hazard (e.g., noise) had relatively higher risks of developing mental health problems than others without such exposure [38].

Workers' psychosocial well-being is not only associated with physical work environment (e.g., work hours, dangerous work environment), but also with psychosocial work environment. In the present study, high job demands and low job autonomy were associated with poor psychological well-being, which is consistent with the prediction of the Demand-Control model (JDC) and other studies $[19,39]$. The French "GAZEL Study" found that compared to low levels of job demands, high levels of job demands increased the odds ratios for poor mental health by 1.8 for men and 1.4 for women; compared to high job autonomy, odds ratio for low job autonomy was 1.4 for both genders [40,41]. However, we didn't find significant association between worksite social support and mental health. Similarly, in van der Doef's review of 232 studies on the Job Demand-Control-Support model and psychological well-being [42], 135 (58\%) studies did not find statistically significant relationship between psychological well-being and worksite social support. Therefore, in order to improve workers' psychological well-being, it may be an effective strategy to reduce job demands and increase workers' job autonomy in workplace.

Previous study found that the depressive rates of the Chinese working population in mild, moderate, and severe status were $25.6 \%, 23.5 \%$, and $1.52 \%$, respectively [9]. Our study also shows high prevalence of poor psychological well-being (35.3\%) in workers in SMEs. Besides researches that reports higher psychological distress levels among women $[28,43]$. There are also literatures reporting men more likely to have worse psychological well-being
[44,45] which is similar to our study. In China, men are mainly responsible for matters outside of the home (which often means source of income), while women are mainly responsible for matters inside of the home (which often means chores at home). Compared with women, men have to confront more social and economic pressure, and prefer to face difficulties alone rather than share the burden. Consistent with previous findings, workers who are older are better in their mental health status as increased knowledge and experiences in work and life may help them better accustomed to environment $[44,45]$. Age, in our study, is also associated with increased job autonomy and decreased job demands which may contribute to better health status among older workers [46]. Psychosocial well-being of migrant workers, as indicated in our study and previous studies, was worse than permanent resident workers [47]. Due to changes in living environment and reduced social network and support, migrant workers often experience more mental health problems than the local residents [47]. Future mental health promotion among factory workers needs to target particularly on migrant workers in SMEs as they are a most vulnerable population subject to hazard working environment.

There are several limitations of this study that should be acknowledged. First, the cross-sectional research design in the current study does not allow causal analyses, only associations. It is plausible that poor mental health may have preceded hazardous work environment (i.e., reverse causation). Associations between physical and psychosocial work-related factors and poor mental health should be further explored in longitudinal studies among SMEs workers in China. Second, we didn't have objective measurements of work environment. All measurements are self-report which may subject to recall bias or socially desired preferences. Third, a wide range of factors may be related to mental health of workers, our study mainly included work-related factors and individual social demographic factors whereas other important factors (e.g., family relationship, social support) that may potentially influence mental health are not included. Fourth, we used a few key indicators (not a complete scale) to determine work-related factors based on the Job Demand-Control-Support (JDCS) model, and Chinese Version of Job Content Questionnaire. Even though all the Cronbach's alpha of work-related factors is above 0.6 in our study, the validity of using such scale still needs to be further confirmed.

\section{Conclusions}

Our study is one of the first to assess psychological wellbeing and examine the associated work environment factors among workers in SMEs in China. The results indicate that the JDCS model is a useful framework in examining psychological well-being among Chinese workers in SMEs. 
The primary policy implication is that young migrant male workers are a key population for mental health promotion. Both physical and psychosocial work environment should be taken into account in policy making to prevent mental illness and promote psychological well-being among workers in SMEs in China.

\section{Competing interests}

The authors declare that they have no competing interests.

\section{Authors' contributions}

ZZ designed the study, the workers survey, and the statistical analyses and drafted the manuscript. YG oversaw the study design and participated in drafting the manuscript. LLu participated in the survey of workers and data analyses. LH and WC participated in the survey of workers. LiL participated in the design of the study and was the study's PI. All authors read and approved the final manuscript.

\section{Authors' information}

Zhi Zeng and Yan Guo: co-first authors.

\section{Acknowledgements}

We are very grateful to the workers interviewed. The author would like to thank the local CDC, the Department of Occupational Disease Prevention and Control, the Department of Health Supervisor and neighborhood offices involved who helped in respondent referral and data collection. Data Access and Responsibility: Zhi Zeng and Li Ling had full access to all the data in the study and take responsibility for the integrity of the data and the accuracy of the data analyses.

This research was funded by the China Medical Board (Grant No. 09-984 and No. 12-111). The funder had no further role in the study design, data collection, analyses or interpretation of the data, writing of the report, or the decision to submit the paper for publication.

Received: 8 May 2014 Accepted: 17 October 2014

Published: 12 November 2014

\section{References}

1. Liu S: The number of SMEs has accounted for more than $99 \%$ of the total of enterprises in China. In 2010. http://news.xinhuanet.com/fortune/ 2010-09/26/___12608785.htm.

2. Luo YY, He JM, Du WJ, Zeng WF, Li JL, Liu YM: Survey on occupational hazards of 58 small industrial enterprises in Guangzhou city. Chinese J Industrial Med 2008, 21:186-187.

3. National Bureau of Statistics of China: National Statistics Yearbook 2012. Beijing: China Statistic Press; 2011.

4. National Bureau of Statistics of China: National report on migrant workers of China. In 2012. http://www.gov.cn/gzdt/2013-05/27/content_2411923.htm.

5. Qiu PY, Yang Y, Wu F, Cao X, Zhao SN, Ma X: Domestic and foreign mental health research progress and enlightenment of migrants (review). Chin Ment Health J 2010, 24:64-68.

6. Peng HJ, Long Y, Liang YX: Mental health status of the SMEs employees in the Pearl River Delta. In The Tenth National Chinese Psychiatric Academic Conference. Nanjing city, Jiangsu Province, China: Jiangsu Medical Association; 2012.

7. Luo $X L$, Huang $L L, M a J H$, Wang $X$ : Mental health status and its influencing factors among workers in the small industrial enterprises in Guangzhou City. Occupation Health 2012, 28:2709-2711.

8. Martin A, Sanderson K, Scott J, Brough P: Promoting mental health in small-medium enterprises: an evaluation of the "Business in Mind" program. BMC Public Health 2009, 9:239.

9. Murray CJ, Lopez AD: Alternative projections of mortality and disability by cause 1990-2020: Global Burden of Disease Study. Lancet 1997, 349:1498-1504.

10. Yang $C$, Li YH, Ma Y, Nie XQ, Pang J, Tao MX, Yang XL: Survey on the psychological health of 5 kinds of urban occupational population and their social support in 6 provinces in China. Chinese J Health Educ 2009, 25:407-410

11. World Health Organization: Strengthening mental health promotion. In 2001. http://who.int/mediacentre/factsheets/fs220/en/.
12. Bech $\mathrm{P}$, Andersen MB, Bech-Andersen $\mathrm{G}$, Tonnesen $\mathrm{S}$, Agnarsdottir E, Borg V: Work-related stressors, depression and quality of life in Danish managers. Eur Psychiatr 2005, 20(Suppl 3):S318-S325.

13. Van Berkel J, Proper KI, van Dam A, Boot CR, Bongers PM, van der Beek AJ: An exploratory study of associations of physical activity with mental health and work engagement. BMC Public Health 2013, 13:558,

14. Li XF, Yang SH, Xu SJ: Investigation of the Mental Health states of the workers in shifts. Med J Chinese People's Health 2006, 18:216-217.

15. Wu SD, Gu XP, Li F, Chen JG: The Analysis of Mental Factors in the Workers of Small-scale Factories. J Environ Occup Med 2002, 19:93-95.

16. Sun BK: Effects of spinning and weaving noise to mental health of workers. J Zhengzhou University (Medical Sciences) 2007, 42:734-737.

17. Zhang MT, Xiao QH, Liang N: Investigation on the mental health in the workers exposed to n-hexane. China Occup Med 2009, 36:222-224. 226.

18. Bonde JP, Munch-Hansen T, Wieclaw J, Westergaard-Nielsen N, Agerbo E: Psychosocial work environment and antidepressant medication: a prospective cohort study. BMC Public Health 2009, 9:262.

19. Karasek RA: Job demands, job decision latitude, and mental strain: Implications for job redesign. Adm Sci Q 1979, 24:285-308.

20. Stansfeld S, Candy B: Psychosocial work environment and mental healtha meta-analytic review. Scand J Work Environ Health 2006, 32:443-462.

21. Jia XD, Jin XP: Status and prevention of organic solvents occupational hazards in China. Chinese J Industrial Hygiene Occup Dis 2000, 18:65-67.

22. Giorgi G, Leon-Perez JM, Cupelli V, Mucci N, Arcangeli G: Do I Just Look Stressed or am I Stressed? Work related Stress in a Sample of Italian Employees. Industrial Health 2014, 52:43-53.

23. World Health Organization: Wellbeing Measures in Primary Health Care: the DepCare Project: Report on a WHO Meeting Stockholm, Sweden 12-13 February 1998. Geneva: WHO Regional Office for Europe; 1998.

24. Bech P, Gudex C, Johansen KS: The WHO (Ten) Well-Being Index: validation in diabetes. Psychother Psychosom 1996, 65:183-190.

25. World Health Organization: Mastering depression in primary care. Frederiksborg General Hospital: World Health Organization, Regional Office for Europe, Psychiatric Research Unit; 1998.

26. Lowe B, Spitzer RL, Grafe K, Kroenke K, Quenter A, Zipfel S, Buchholz C, Witte $\mathrm{S}$, Herzog W: Comparative validity of three screening questionnaires for DSM-IV depressive disorders and physicians' diagnoses. J Affect Disord 2004, 78:131-140.

27. Henkel V, Mergl R, Kohnen R, Allgaier AK, Moller HJ, Hegerl U: Use of brief depression screening tools in primary care: consideration of heterogeneity in performance in different patient groups. Gen Hosp Psychiatr 2004, 26:190-198.

28. Giorgi G, Ando M, Arenas A, Shoss MK: Exploring Personal and Organizational Determinants of Workplace Bullying and Its Prevalence in a Japanese Sample. Psychol Violence 2012, 3:185-197.

29. Awata S, Bech P, Yoshida S, Hirai M, Suzuki S, Yamashita M, Ohara A, Hinokio Y, Matsuoka H, Oka Y: Reliability and validity of the Japanese version of the World Health Organization-Five Well-Being Index in the context of detecting depression in diabetic patients. Psychiatry Clin Neurosci 2007, 61:112-119.

30. World Health Organization: Wellbeing Measures in Primary Health Care: The DepCare Project. Copenhagen: WHO, Regional Office for Europe; 1998.

31. Li J, Yang WJ, Liu P, Xu ZF, Cho S: Psychometric Evaluation of the Chinese (Mainland) Version of Job Content Questionnaire: A Study in University Hospitals. Indust Health 2004, 42:260-267.

32. An SL, Chen PY: Measurement Scales: Reliability and Affecting Factors, Chinese J Clin Psychol 2001, 9:315-318.

33. National Bureau of Statistics of China: National Statistics Yearbook 2012 Beijing: China Statistic Press; 2012.

34. Mausner-Dorsch H, Eaton WW: Psychosocial work environment and depression: epidemiologic assessment of the demand-control model. Am J Public Health 2000, 90:1765.

35. Parkes KR, Von Rabenau C: Work characteristics and well-being among psychiatric health-care staff. I Community Appl Soc Psychol 1993, 3:243-259.

36. Amagasa T, Nakayama T: Relationship between long work hours and depression in two working populations: a structural equation model approach. J Occup Environ Med 2012, 54:868-874.

37. Dragano N, He Y, Moesbus S, Jöckel K-H, Erbel R, Siegrist J: Two models of job stress and depressive symptoms. Soc Psychiatr Psychiatr Epidemiol 2008, 43:72-78.

38. Zhang HX, Zhang XB, Tian T: The factors affecting coal mine workers' mental health in Social transformation period. J Qiqihar Med Coll 2010, $31: 3778-3780$. 
39. Kawakami N, Araki S, Kawashima M: Effects of job stress on occurrence of major depression in Japanese industry: a case-control study nested in a cohort study. J Occup Med 1990, 32:722-725.

40. Paterniti S, Niedhammer I, Lang T, Consoli SM: Psychosocial factors at work, personality traits and depressive symptoms. Longitudinal results from the GAZEL Study. Br J Psychiatry 2002, 181:111-117.

41. Niedhammer I, Bugel I, Goldberg M, Leclerc A, Gueguen A: Psychosocial factors at work and sickness absence in the Gazel cohort: a prospective study. Occup Environ Med 1998, 55:735-741.

42. Van der Doef M, Maes S: The job demand-control (-support) model and psychological well-being: a review of 20 years of empirical research. Work Stress 1999, 13:87-114.

43. Magnavita N, Fileni A, Magnavita G: Work stress in radiologists-A pilot study. Radiol Med 2008, 113:329-346.

44. Maccoby EE, Jacklin CN: The Psychology of Sex Differences. California: Stanford University Press; 1974.

45. Yuan H: Socioeconomic Status, Age and Mental Health: An Empirical Study in Shanghai. Society 2011, 31:159-182.

46. Magnavita N: Workplace violence and occupational stress in healthcare workers: a chicken-and-egg situation-results of a 6-year follow-up study. J Nurs Scholarsh 2014, 46:366-376.

47. Wang GX, Su XX: Social Support/Stress and Impacts on Physical and Mental Health: Comparison between Shanghai Migrants and Local Residents. Popul Dev 2011, 17:2-9.

doi:10.1186/1471-2458-14-1162

Cite this article as: Zeng et al:: Mental health status and work environment among workers in small- and medium-sized enterprises in Guangdong, China-a cross-sectional survey. BMC Public Health 2014 14:1162.

\section{Submit your next manuscript to BioMed Central and take full advantage of:}

- Convenient online submission

- Thorough peer review

- No space constraints or color figure charges

- Immediate publication on acceptance

- Inclusion in PubMed, CAS, Scopus and Google Scholar

- Research which is freely available for redistribution 